Supporting information for Bioconjugate Chemistry

\title{
Intracellular uptake mechanism of bioorthogonally conjugated nanoparticles on metabolically engineered mesenchymal stem cells
}

Seungho Lim ${ }^{1,2 \dagger}$, Woojun $\mathrm{Kim}^{2,3 \dagger}$, Sukyung Song ${ }^{2}$, Man Kyu Shim², Hong Yeol Yoon², ByungSoo Kim${ }^{1}$, Ick Chan Kwon ${ }^{2,3}$, and Kwangmeyung Kim${ }^{2,3 *}$

${ }^{1}$ School of Chemical and Biological Engineering, Seoul National University, 1, Gwanak-ro, Gwanak-gu, Seoul, 08826, Republic of Korea

${ }^{2}$ Center for Theragnosis, Biomedical Research Institute, Korea Institute of Science and Technology (KIST), 5, Hwarang-ro 14-gil, Seongbuk-gu, Seoul, 02792, Republic of Korea ${ }^{3}$ KU-KIST Graduate School of Converging Science and Technology, Korea University, 145, Anam-ro, Seongbuk-gu, Seoul, 02841, Republic of Korea

These authors contributed equally to this work

*Corresponding author: (E-mail) kim@kist.re.kr (K.K) 


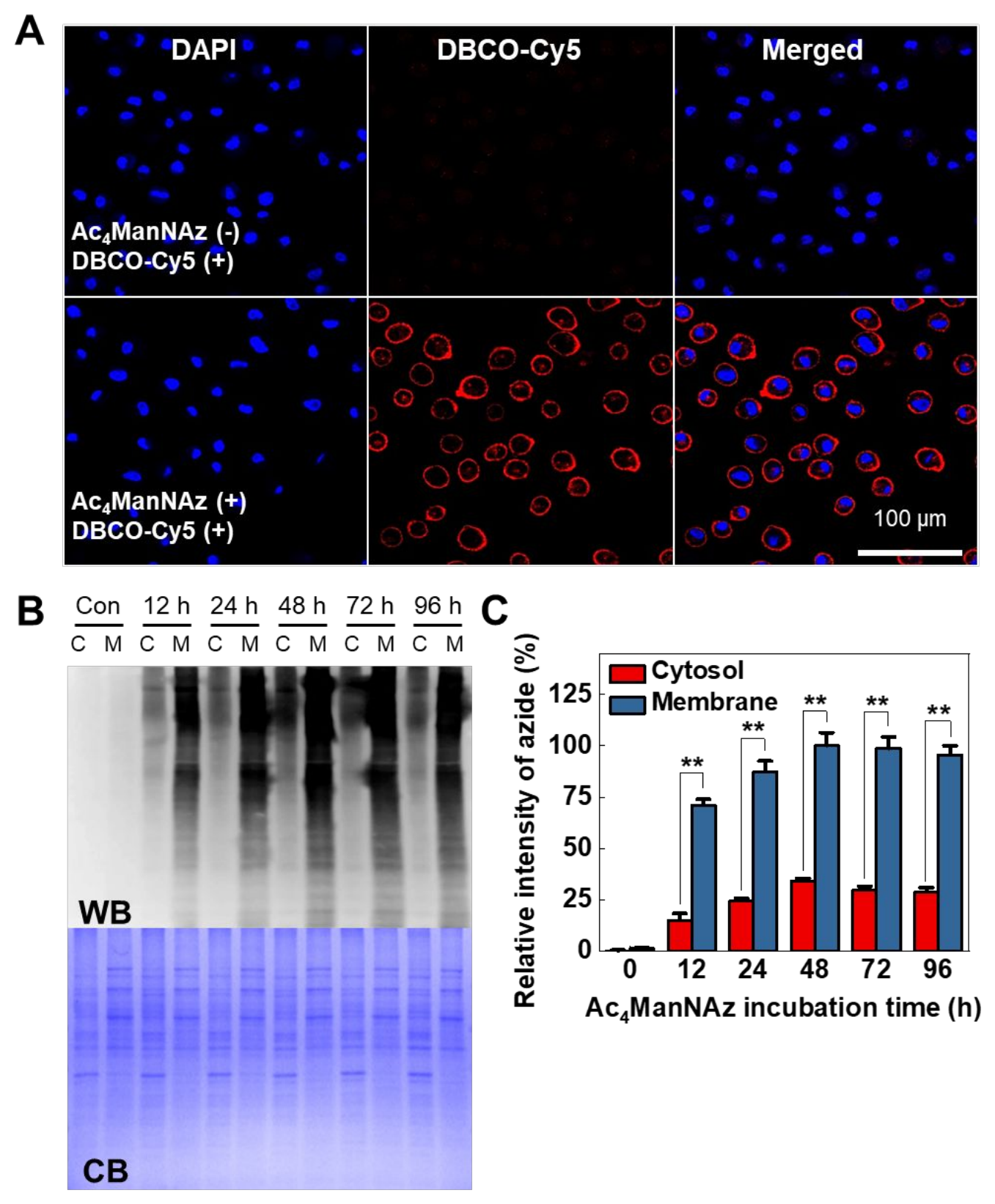

Fig. S1. (A) Confocal images of suspended hMSCs which were treated with $10 \mu \mathrm{M}$ of DBCO-Cy5. To generate azide groups on the cell surface $10 \mu \mathrm{M}$ of $\mathrm{Ac}_{4} \mathrm{ManNAz}$ was pretreated for $48 \mathrm{~h}$. As a control, hMSCs were treated with $10 \mu \mathrm{M}$ of DBCO-Cy5 without $\mathrm{Ac}_{4}$ ManNAz-treatment. (B) Western blot analysis (WB) and Coomassie Blue staining (CB) of $\mathrm{Ac}_{4}$ ManNAz-treated hMSCs at different time points (12, 24, 48, 72, and 96h). Azide groups of each cell at different time points were extracted from cytoplasmic protein (abbreviation: C) and membrane protein (abbreviation: M) (C) Relative intensity of each azide band of (A) western blot analysis. $(* *)$ indicate difference at the $p<0.01$. 


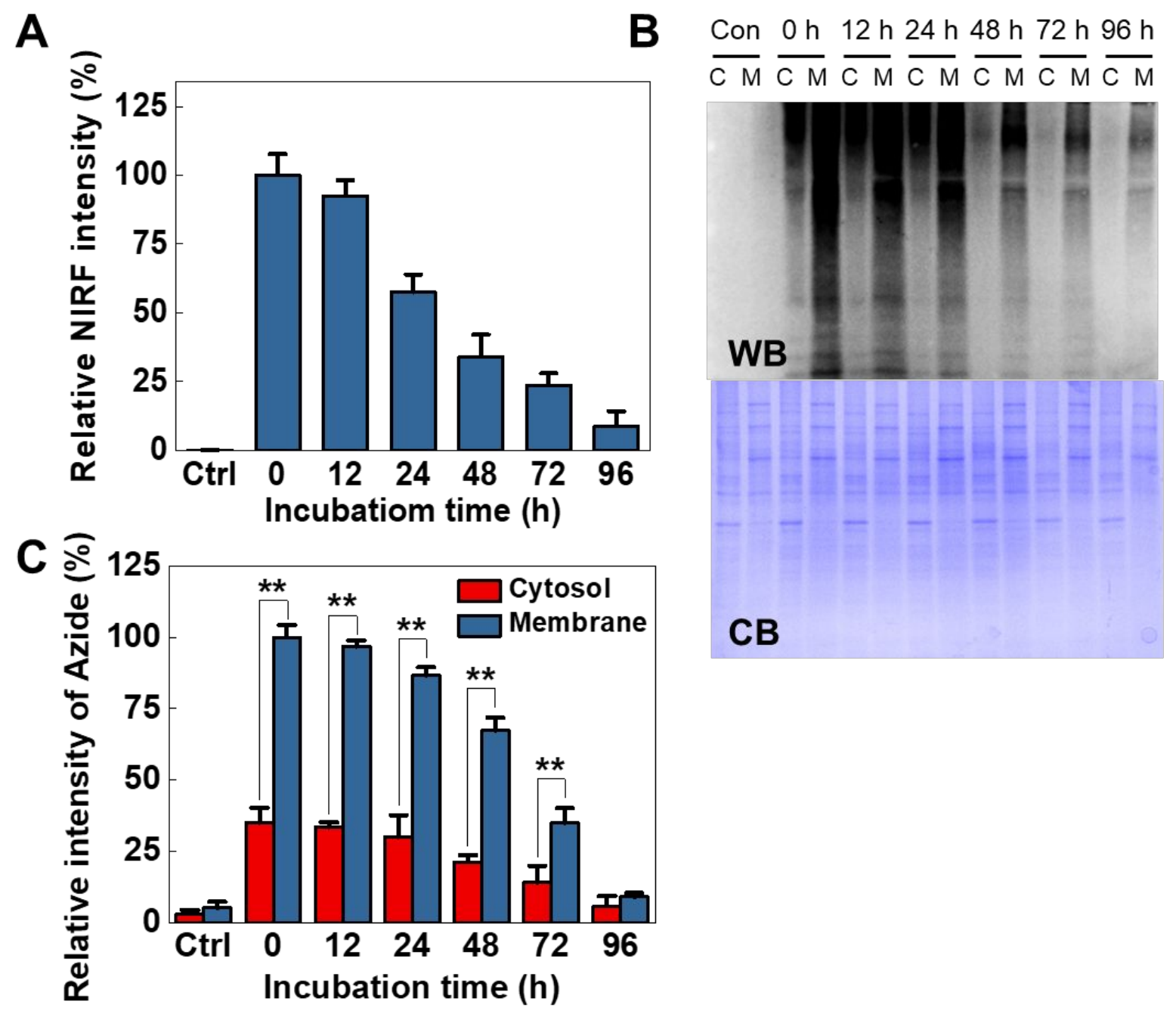

Fig. S2. (A) Relative time-dependent NIRF intensity of DBCO-Cy5 on $\mathrm{Ac}_{4} \mathrm{ManNAz}$-treated hMSCs at different further incubation times from $0 \mathrm{~h}$ to 96. (B) Western blot analysis (WB) and Coomassie Blue staining (CB) of azide groups from the cytoplasmic protein (abbreviation: C) and membrane protein (abbreviation: M) of hMSCs at various time points after $10 \mathrm{uM} \mathrm{Ac}_{4} \mathrm{ManNAz}$ treatment. (C) Relative intensity of each azide band of (B) western blot analysis. (**) indicate difference at the $p<0.01$. 
A

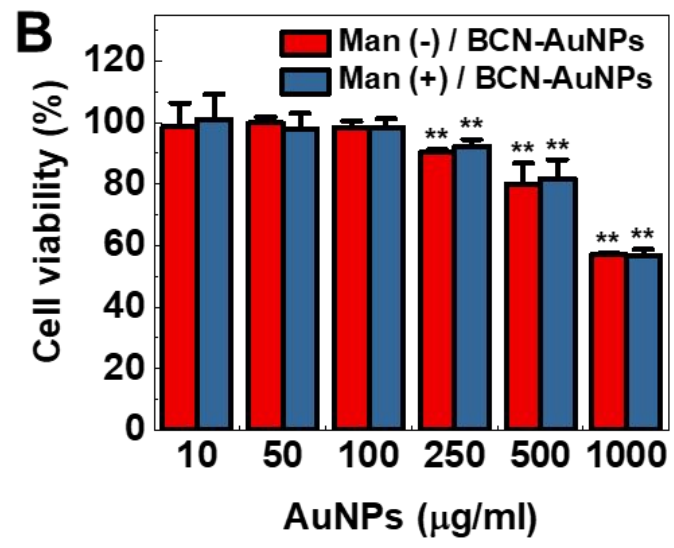

Fig. S3. (A) NIRF signal from BCN-AuNPs and unmodified AuNPs. (B) Cell viability of $\mathrm{Ac}_{4} \mathrm{ManNAz}$-treated and untreated hMSCs after $12 \mathrm{~h}$ post-incubation of various concentrations of BCN-AuNPs $(10-1000 \mu \mathrm{g} / \mathrm{mL})$.

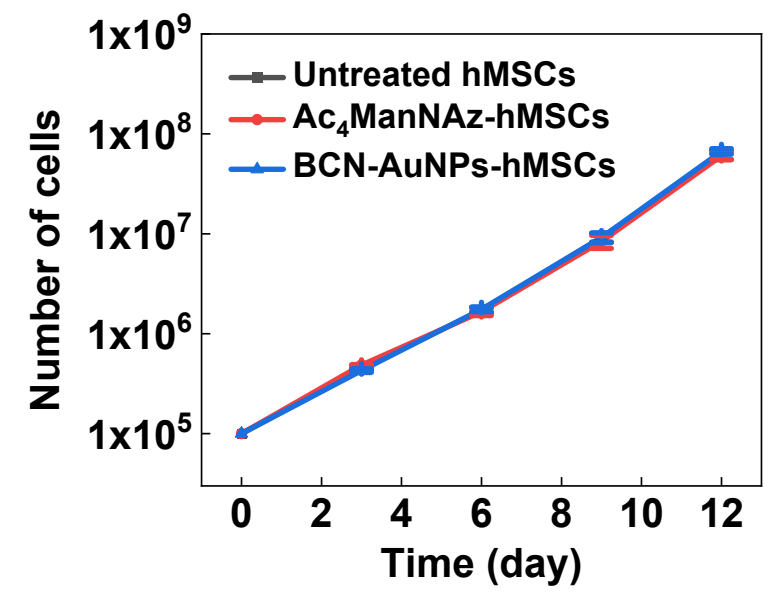

Fig. S4. Proliferation rate of untreated hMSCs (Untreated hMSCs), $10 \mu \mathrm{M}$ of $\mathrm{Ac}_{4} \mathrm{ManNAz}$ treated hMSCs ( $\mathrm{Ac}_{4} \mathrm{ManNAz-hMSCs}$ ), and $10 \mu \mathrm{M}$ of $\mathrm{Ac}_{4} \mathrm{ManNAz}$ and $100 \mu \mathrm{g}$ of BCNAuNPs-treated hMSCs (BCN-AuNPs-hMSCs) over 12 days. 